\title{
Wideband waveform design based on spectrally constrained optimization
}

\author{
Shunhong $\mathrm{Li}^{1, \mathrm{a}}$, Tengda Pei $\mathrm{i}^{2, \mathrm{~b}}$ \\ ${ }^{1}$ College of Information Engineering, Dalian University, Dalian 116622, China; \\ ${ }^{2}$ College of Information Engineering, Dalian University, Dalian 116622, China. \\ a263673794@qq.com, b2109863677@qq.com
}

Keywords: Wideband pulse; Waveform design; Spectrum notch; Spectrum shape

\begin{abstract}
An improved spectrum shaping iterative algorithm is proposed in this paper in order to solve a wideband waveform design. The spectrally constrained condition is set up before an optimally initial signal is obtained by constraining the amplitude spectrum of the input initial signal. With which, an existing iterative process is initialized on the basis of spectral constraint, the desired signal would be output when the terminate condition of the process is satisfied. The simulation results based on a wideband LFM signal has shown that in the spectrum notch depth range of $30-40 \mathrm{~dB}$, the iteration number and time performance of the improved algorithm are significantly better than the original, with it having more outstanding robustness.
\end{abstract}

\section{Introduction}

RF spectrum is a kind of limited natural resources, and a large number of wireless applications, such as the fixed, mobile, broadcasting, amateur, space research, meteorology, global positioning system (GPS), environmental monitoring, and the communication services that guarantee the safety of maritime and air traffic, etc., have constantly been increasing on the spectrum demand. While a spectrum monitoring experiment in Hoboken river of New Jersey for $700 \sim 800 \mathrm{MHz}$ frequency band claimed $^{[1]}$, part of the spectrum was always used, and part of that never used, in addition the other part used in some period of time. To some extent this work has revealed one fact that the spectrum resources are not sufficiently utilized, in contrast there is a certain waste. Therefore, in the wideband waveform design, incorporating notches into some specific bands ${ }^{[2]}$ in order to avoid mutual interference, achieving spectrum sharing, and efficiently taking advantage of spectrum resources seem valuable a lot.

The US Federal Communications Commission (FCC) released in 2002 the provision of spectral masks (spectral boundary) ${ }^{[3]}$ so as to make the spectrum resources the most efficiently and rationally used of, which not only limits the power spectral density(PSD) of the wideband signal to a very low level, but also sets a different PSD for different frequency bands to prevent various wireless wideband signals in the same band from mutually interfering, moreover, typically requires radar signal attenuated by more than $30 \mathrm{~dB}$ at the bands allocated to the other systems, i.e., the spectrum notch depth have to reach or exceed $30 \mathrm{~dB}$.

So far, a large number of broadband waveform design achievements has emerged here based on FCC spectral masks ${ }^{[4-9]}$. Literature[4] used the orthogonal Hermite-Rodriguez basis functions and linear FM(LFM) to model the design problem as a quadratic semi-infinite programming one, resulting in fine effect with being too complicated; Paper[5] utilized Remez(Parks-McClellan) algorithm to take spectral constraint and shaping Based on Chebyshev approximation method, which is actually performing FIR filtering, simple and easy to implement, but can not optimize spectrum utilization and easily reach expected spectrum notch. [6] derived the spectral notches from optimizing the signal autocorrelation function, which can not be capable of controlling accurately the width and depth of the spectrum notches; [9] proposed a simple and efficient iterative algorithm that it reaches design targets by constraining the amplitude of the domain sequence and spectrum, however, is 
sensitive and dependent to and on the initial input signal. Based on the literature [9], an improved algorithm was proposed, the relevant simulation and verification was implemented as well.

\section{The improved method}

The waveform design issue in [9] can be expressed:

$$
\begin{aligned}
& \min _{\mathbf{x}, \varphi, \mathbf{z}}\|\mathbf{F x}-\varphi \mathbf{z}\|_{2}^{2} \\
& \text { s.t. } \\
& \quad\left|x_{i}\right|^{2}=q_{i}, i=1,2, \ldots, N \\
& \quad f_{i} \leq\left|z_{i}\right| \leq g_{i}, i=1,2, \ldots, N
\end{aligned}
$$

Where $\varphi$ is a complex scalar, $\mathbf{z} \in \mathbb{C}^{N \times 1}$ is the spectrum of desired signal, and $\mathbf{x} \in \mathbb{C}^{N \times 1}$ is the time-domain signal sequence, $\mathbf{F} \in \mathbb{C}^{N \times N}$ is an Unitary Fourier matrix, $\|\cdot\|_{2}$ denotes the Euclidean Norm of one vector. $\mathbf{q} \in \mathbb{R}^{N \times 1}$ denotes the amplitude constraint vector of sequence $\mathbf{x} . \mathbf{f}, \mathbf{g} \in \mathbb{R}^{N \times 1}$ represents the boundary range of spectrum $\mathbf{z}$ from up and down, respectively.

The solution of the model in (1) can be carried by using the iterative method as follows: 1) input parameters $(\mathbf{x}, \varphi)$ to acquire the target spectrum $\hat{\mathbf{z}} ; 2)$ use $(\mathbf{x}, \hat{\mathbf{z}})$ to evaluate $\hat{\varphi} ; 3)$ utilize $(\hat{\mathbf{z}}, \hat{\varphi})$ to obtain $\hat{\mathbf{x}}$. Step 1) - 3) are repeated until the algorithm meets the terminate or convergence condition. However, it is difficult for the optimal $\mathbf{x}^{0}$ to be determined, which has a great impact on convergence performance of the algorithm. Based on the problem above, in this paper, a preprocessing model is set up in front of the model in (1). In advance, the amplitude spectrum of initial signal was optimized and constrained to the scope of $\boldsymbol{\alpha}, \boldsymbol{\beta} \in \mathbb{R}^{N \times 1}$ with $(\mathbf{x}, \boldsymbol{\mu})$ regarded as the input of the preprocessing, resulting in the optimally initial signal spectrum $\hat{\mathbf{h}}$ which satisfies the desired amplitude spectrum constraint condition $\mathbf{f}, \mathbf{g}$. Therefore, initializing the model in (1) with $(\hat{\mathbf{h}}, \varphi)$ are easier causing the algorithm converging. The preprocessing model like as (2).

$$
\begin{aligned}
& \min _{\mathbf{x}, \mathbf{h}, \boldsymbol{\mu}}\|\mathbf{F} \mathbf{x}-\boldsymbol{\mu} \odot \mathbf{h}\|_{2}^{2} \\
& \text { s.t. } \alpha_{i} \leq\left|h_{i}\right| \leq \beta_{i}, i=1,2, \ldots, N
\end{aligned}
$$

Where, $\alpha_{i}$ and $\beta_{i}$ meet $\alpha_{i}<\beta_{i}$, the scope defined by $\boldsymbol{\alpha}, \boldsymbol{\beta}$ is narrower than that defined by $\mathbf{f}, \mathbf{g}$. $\odot$ denotes the element-wise product operation between vectors, $\boldsymbol{\mu} \in \mathbb{C}^{N \times 1}$ is complex modulus factor vector, $\mathbf{h} \in \mathbb{C}^{N \times 1}$ is the optimally initialization signal spectrum wanted. The preprocessing model like as follows :

1 ) input the initial signal $\mathbf{x}^{0}$, the initial modulus factor vector $\boldsymbol{\mu}^{0}$, solve the constraint and optimization problem with respect to $\mathbf{h}$ :

$$
\begin{aligned}
& \min _{\mathbf{h}}\|\mathbf{F x}-\boldsymbol{\mu} \odot \mathbf{h}\|_{2}^{2} \\
& \text { s.t. } \alpha_{i} \leq\left|h_{i}\right| \leq \beta_{i}, i=1,2, \ldots, N
\end{aligned}
$$

The cost function in (3) can be recasted as :

$$
\begin{aligned}
f_{\mathbf{x}, \boldsymbol{\mu}}(\mathbf{h}) & =(\mathbf{F x}-\mathbf{\kappa})^{\mathrm{H}}(\mathbf{F} \mathbf{x}-\mathbf{\kappa}) \\
& =(\mathbf{F x})^{\mathrm{H}}(\mathbf{F x})+\boldsymbol{\kappa}^{\mathrm{H}} \mathbf{\kappa}-2 \mathfrak{R} e\left\{(\mathbf{F x})^{\mathrm{H}} \mathbf{\kappa}\right\} \\
& =\tilde{\mathbf{x}}^{\mathrm{H}} \tilde{\mathbf{x}}+\boldsymbol{\kappa}^{\mathrm{H}} \mathbf{\kappa}-2 \mathfrak{R} e\left\{\tilde{\mathbf{x}}^{\mathrm{H}} \mathbf{\kappa}\right\}
\end{aligned}
$$

where $\boldsymbol{\kappa}=\boldsymbol{\mu} \odot \mathbf{h}, \tilde{\mathbf{x}}=\mathbf{F x}$. Further derivation of (4) leads to :

$$
f\left(\tilde{x}_{i}, \kappa_{i}\right)=\sum_{i=1}^{N}\left[\left|\tilde{x}_{i}\right|^{2}+\left|\kappa_{i}\right|^{2}-2\left|\tilde{x}_{i} \| \kappa_{i}\right| \cos \left(\phi_{\tilde{x}_{i}}-\phi_{\kappa_{i}}\right)\right]
$$


To make $f\left(\tilde{x}_{i}, \kappa_{i}\right)$ a minimum value, $\cos \left(\phi_{\tilde{x}_{i}}-\phi_{\kappa_{i}}\right)=1$ shall be established, simultaneously, the angle relationship between $\tilde{x}_{i}$ and $\kappa_{i}$ meets $\phi_{\tilde{x}_{i}}=\phi_{\kappa_{i}}=\phi_{\mu_{i}}+\phi_{h_{i}}$. Then the formula (5) is equivalent to :

$$
\begin{aligned}
f_{\tilde{x}_{i}, \mu_{i}}\left(h_{i} \mid \phi_{\tilde{x}_{i}}=\phi_{\kappa_{i}}\right) & =\sum_{i=1}^{N}\left[\left(\left|\tilde{x}_{i}\right|-\left|\kappa_{i}\right|\right)^{2}\right] \\
& =\sum_{i=1}^{N}\left[\left(\left|\tilde{x}_{i}\right|-\left|\mu_{i}\right| \cdot\left|h_{i}\right|\right)^{2}\right]
\end{aligned}
$$

In fact, Minimizing (4) is equivalent to Minimizing (6) that is quadratic function with respect to $h_{i}$, the solution can be accessed easily :

$$
\hat{h}_{i}=\left\{\begin{array}{l}
\beta_{i} \mathrm{e}^{\mathrm{j}\left(\phi_{x_{i}}-\phi_{\mu_{i}}\right)},\left|\tilde{x}_{i}\right| /\left|\mu_{i}\right|>\beta_{i} \\
\alpha_{i} \mathrm{e}^{\mathrm{j}\left(\phi_{x_{i}}-\phi_{\mu_{i}}\right)},\left|\tilde{x}_{i}\right| /\left|\mu_{i}\right|<\alpha_{i} \\
\frac{\tilde{x}_{i}}{\mu_{i}}, \text { others } \\
\quad i=1,2, \ldots, N .
\end{array}\right.
$$

In actual operation of the algorithm, $\left|h_{i}\right|$ would oscillate around the frequency boundary $\alpha_{i}$ and $\beta_{i}$, which makes the constraint $\alpha_{i} \leq\left|h_{i}\right| \leq \beta_{i}$ difficult to fully implement. So making a proper relaxation on the solution demostrated by (7) brings about the optimal solution $\hat{\mathbf{h}}$ :

$$
\hat{h}_{i}=\left\{\begin{array}{l}
\left(\beta_{i}-\varepsilon\right) \mathrm{e}^{\mathrm{j}\left(\phi_{\tilde{x}_{i}}-\phi_{\mu_{i}}\right)},\left|\tilde{x}_{i}\right| /\left|\mu_{i}\right|>\beta_{i} \\
\left(\alpha_{i}+\varepsilon\right) \mathrm{e}^{\mathrm{j}\left(\phi_{x_{i}}-\phi_{\mu_{i}}\right)},\left|\tilde{x}_{i}\right| /\left|\mu_{i}\right|<\alpha_{i} \\
\frac{\tilde{x}_{i}}{\mu_{i}}, \text { others } \\
i=1,2, \ldots, N .
\end{array}\right.
$$

where, $\varepsilon>0$ is a little value that denotes the relaxation level.

2) use $\hat{\mathbf{h}}$ and $\boldsymbol{\mu}$ to calculate $\mathbf{x}$ :

$$
\min _{\mathbf{x}}\|\mathbf{F x}-\boldsymbol{\mu} \odot \mathbf{h}\|_{2}^{2}
$$

3) use $\hat{\mathbf{h}}$ and $\hat{\mathbf{x}}$ to calculate $\boldsymbol{\mu}$ :

$$
\min _{\boldsymbol{\mu}}\|\mathbf{F x}-\boldsymbol{\mu} \odot \mathbf{h}\|_{2}^{2}
$$

Since the solving in step 2) and 3) isn't restrained at all, the solutions would be respectively derived $: \hat{\mathbf{x}}=\mathbf{F}^{\mathrm{H}}(\hat{\mathbf{h}} \odot \boldsymbol{\mu}), \hat{\mu}_{i}=\frac{\bar{x}_{i}}{\hat{h}_{i}}$, where $\overline{\mathbf{x}}=\mathbf{F} \hat{\mathbf{x}}=\left[\bar{x}_{1}, \bar{x}_{2}, \ldots, \bar{x}_{N}\right]^{\mathrm{T}}$.

The preprocessing algorithm above can be expressed in the following pseudocodes :

Initialize:

1) Set $\boldsymbol{\mu}^{0}=\mathbf{1}_{N}, \varepsilon>0$

2) Generate the Initial sequence $\mathbf{x}^{0}$

3) Set the max iteration $K$ and the iteration index $k=1$

4) Let temp variable $\mathbf{t}^{1}=\mathbf{F} \mathbf{x}^{0} . / \boldsymbol{\mu}^{0}$

Repeat

\{ for $i=1$ to $N$

1) Calculate $\mathbf{h}^{k}$ using $\mathbf{x}^{k-1}, \boldsymbol{\mu}^{k-1}$ 
if $\left|t_{i}^{k}\right|>\beta_{i}$ then

$$
h_{i}^{k}=\left(\beta_{i}-\varepsilon\right) t_{i}^{k} /\left|t_{i}^{k}\right|
$$

else if $\left|t_{i}^{k}\right|<\alpha_{i}$ then

$$
h_{i}^{k}=\left(\alpha_{i}+\varepsilon\right) t_{i}^{k} /\left|t_{i}^{k}\right|
$$

else

$$
h_{i}^{k}=t_{i}^{k}
$$

end if

2) Calculate $\mathbf{x}^{k}$ using $\mathbf{h}^{k}, \boldsymbol{\mu}^{k-1}$ as $\mathbf{x}^{k}=\mathbf{F}^{\mathrm{H}}\left(\mathbf{h}^{k} \odot \boldsymbol{\mu}^{k-1}\right)$

3) Calculate $\boldsymbol{\mu}^{k}$ using $\mathbf{x}^{k}, \mathbf{h}^{k}$ as $\boldsymbol{\mu}^{k}=\mathbf{F} \mathbf{x}^{k} . / \mathbf{h}^{k}$

4) Let $\mathbf{t}^{k}=\mathbf{F} \mathbf{x}^{k}$./ $\boldsymbol{\mu}^{k}$

5) Let $k=k+1$

until $f_{i} \leq\left|t_{i}^{k}\right| \leq g_{i}$ or $k>K$

end for

\section{Simulation}

Designing a LFM signal for wideband radar. Assuming its frequency range differ from 85 to $428 \mathrm{MHz}$ with the time domain pulse width $53.031 \mu$ s and FM rate $6.438(\mathrm{MHz} / \mu \mathrm{s})$, in which four bands 167.2 - 175.8, 233.8 - 294.3, 309.1 - 340.4, and 378.2 - $388 \mathrm{MHz}$ are occupied by four communication radio stations respectively. In order to prevent the radar signal from jamming the radio station, the desired waveform should be provided with four spectrum notches, the depth of these notches reach up to $35 \mathrm{~dB}$. The spectrum of the initial signal $\mathbf{x}^{0}$ is shown in figure (a).

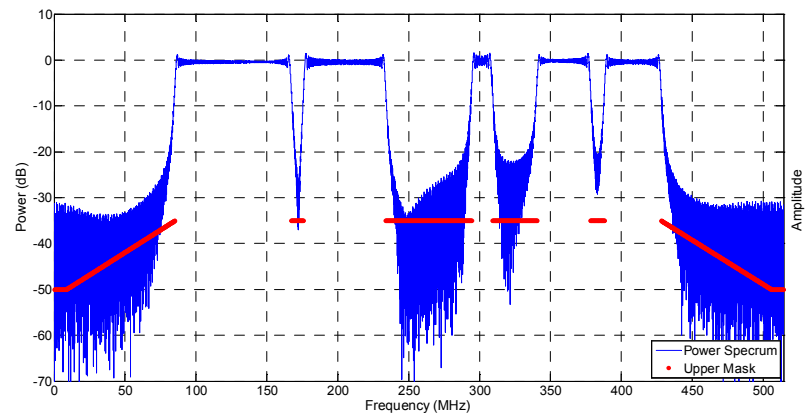

(a) Spectrum of Initial Signal

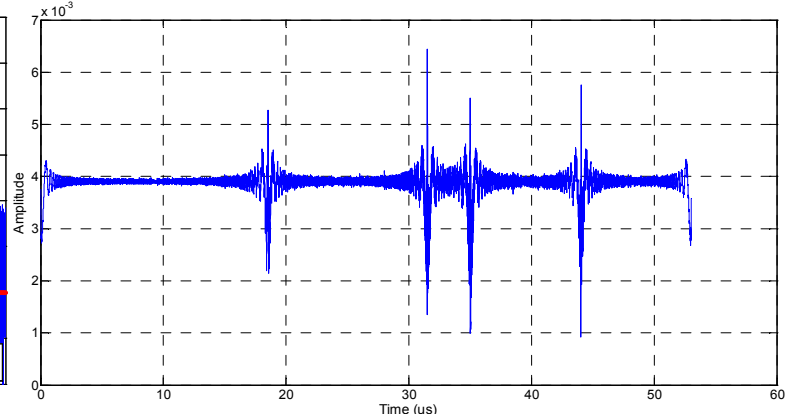

(b) Plot of Optimally Initial Signal

From the graph (a), it's seen that any spectrum notches of the initial signal are not strictly satisfing the requirements of the depth of $35 \mathrm{~dB}$, the improved algorithm was attended to process the input signal. And, respectively, figure (b) and (c) demonstrate the plot and spectrum of the optimally initial signal. From the graph (d) it can be seen that the depth of the four notches in the desired spectrum have strictly reached $35 \mathrm{~dB}$.

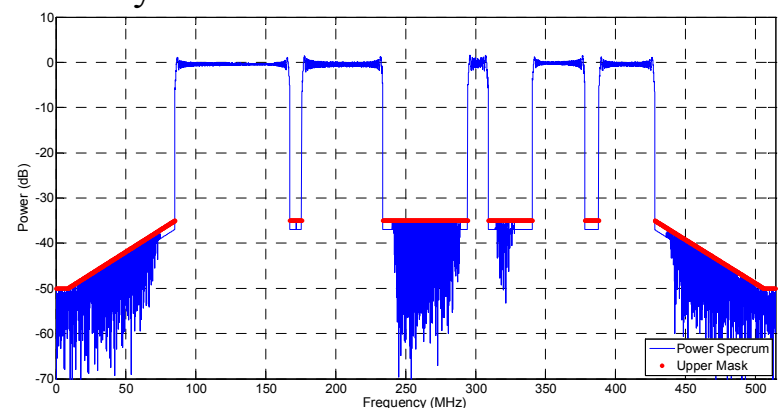

(c) Spectrum of Optimally Initial Signal



(d) Spectrum of Desired Signal

In order to verify the robustness and reliability of the algorithm, as well as the performance of iteration number and the iteration time of the algorithm, the depth of the spectrum notch ranged from 
30 to $40 \mathrm{~dB}$, and the Monte Carlo experiment for 500 times every $2 \mathrm{~dB}$ were performed, as result, the performance of iteration number and the iteration time of the algorithm were shown in the (e) and (f).

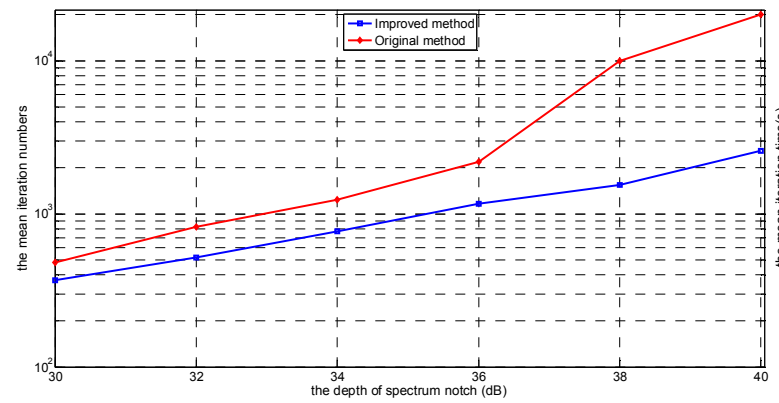

(e) Iteration Number Performance

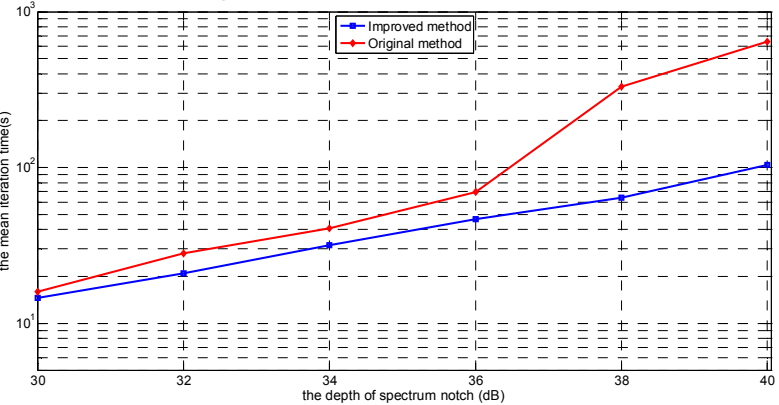

(f) Iteration Time Performance

As can be seen from the graph (e) and (f), in the depth range $30-40 \mathrm{~dB}$, the performance of the improved algorithm is obviously better than that of the original in terms of the number and time of iteration, with the former having better robustness.

\section{Conclusion}

The improved algorithm proposed in this paper is based on the spectrum's constraint and optimization of input signal, in accordance with the design requirements, the spectrum notches are incorporated into the desired signal bands for achieving waveform design. Its characteristics are in that preprocessing the amplitude spectrum of initial input signal to obtain the optimal initialization spectrum, and further to initialize an existing iterative process, so as to achieve the desired waveform. Although the proposed algorithm showed a clear advantage, the flexibility in setting up the width and depth of spectrum notches are still inadequate, in addition, the performance in iteration number and time contains further room for improvement. Finding out a more flexible and efficient algorithm for wideband waveform design is the focus of the next research.

\section{References}

[1] Bruce A. Fette, Cognitive Radio Technology[M]. Burlington: ELSEVIER INC, 2009: 29 - 30.

[2] ZHU Li-ping, ZHU yi-sheng. Waveform Design and Spectrum Shaping Techniques for Cognitive Ultra Wideband Radio [J]. Communications Technology, 2009, 07(42): 48 - 49.

[3] Federal Communications Commission (FCC): 'Revision of part 15 of the commission' s rules regarding ultra-wideband transmission systems' (ET Docket, 2002).

[4] M.A. Yusoff, Z. Zang and S.S. Abeysekera, "Design of spectrally efficient ultra-wideband waveforms using Hermite-Rodriguez functions,” IET Com., vol. 6, pp. 1163 - 1169, July. 2012.

[5] HAO Run-fang, LI Hong-yan, WANG Hua-kui. An UWB Pulse Design Method Based on Chebyshev Function Approximation [J]. COMPUTER SIMULATION, 2008, 25(4): 305 - 307.

[6] M. Lindenfeld, "Sparse frequency transmit and receive waveform design," IEEE Trans. Aerospace Electron. Syst., vol. 40, pp. 851 - 861, July. 2004.

[7] LI Zhan-min, ZHOU Yin, SUN Xue-bin, ZHOU Zheng. A GA-based UWB Pulse Waveform Design [J]. Radio Engineering, 2012, 42(1): 14 - 16.

[8] C. Nunn and L. Moyer, “Spectrally-compliant waveforms for wideband radar," IEEE Aerospace Electron. Syst. Mag., vol. 27, pp. 11 - 15, Aug. 2012.

[9] William Rowe, Petre Stoica and Jian Li, “Spectrally Constrained Waveform Design, ”IEEE Signal Processing Mag., vol. 31, pp. 157 - 162, May. 2014. 